\title{
Central cord syndrome: functional outcome after rehabilitation
}

\author{
AM P-E Tow and KH Kong \\ Department of Rehabilitation Medicine, Tan Tock Seng Hospital, c/o Ang Mo Kio Community Hospital, 17 Ang Mo \\ Kio Avenue 9, Singapore 569766
}

\begin{abstract}
Seventy-three spinal cord injured patients with central cord syndrome who had undergone inpatient rehabilitation, were studied retrospectively with regard to their demographic, neurologic and functional characteristics. There were 67 males and six females with a mean age of 53.5 years. Falls was the commonest mechanism of injury $(54.8 \%)$ followed by motor vehicle accidents. Eleven patients sustained cervical fractures and 41 had radiological evidence of cervical spondylosis. Seventeen patients had sensory impairment and significant spasticity was present in 14 patients. Significant improvements in the admission/discharge ASIA motor scores and Modified Barthel Index $(\mathrm{MBI})$ scores $(P<0.001)$ were noted after rehabilitation. Ninety-two percent of patients were continent of bladder on discharge compared to $64.4 \%$ on admission. Multiple regression analysis revealed three factors associated with a better functional outcome, namely, higher admission MBI scores, absence of spasticity and younger age $(P<0.05)$.
\end{abstract}

Keywords: central cord syndrome; cervical spondylosis; spasticity; rehabilitation

\section{Introduction}

The central cord syndrome (CCS) is the commonest of the incomplete traumatic cervical cord syndromes with the reported incidence varying from $15.7-25 \% .{ }^{1,2}$ First described by Schneider in 1954, it is characterised by disproportionately more motor impairment in the upper than the lower extremities, bladder dysfunction, and varying degrees of sensory loss below the level of lesion. ${ }^{3}$ It is generally reported to have a good prognosis for neurologic and functional recovery. ${ }^{1}$

It has typically been described to occur most commonly in older persons with cervical spondylosis and hyperextension injuries, without apparent damage to the bony spine, ${ }^{4}$ although it may occur at any age with other etiologies, injury mechanisms or predisposing factors. ${ }^{1,5,6}$ Postulated mechanisms include squeezing or pinching of the spinal cord both anteriorly and posteriorly, with inward bulging of the ligamentum flavum during hyperextension.

Favourable long term prognostic factors that have been previously documented include: good hand function, ${ }^{1}$ evidence of early motor recovery, ${ }^{8,9}$ young age, ${ }^{5,6}$ absence of lower extremity neurologic motor impairment at rehabilitation admission, and presence of documented upper and lower extremity strength improvement during rehabilitation. ${ }^{5}$

The purpose of this study is: (1) to examine the demographic, epidemiological and neurologic characteristics and functional outcome achieved by CCS patients participating in a comprehensive inpatient

Correspondence: A-P Tow rehabilitation programme; (2) to further identify factors which are associated with favourable discharge rehabilitation outcomes.

\section{Procedures and methods}

The acute admission and rehabilitation records of all patients with CCS who completed inpatient rehabilitation at the Department of Rehabilitation Medicine, Tan Tock Seng Hospital (TTSH), Singapore, between January 1982 and September 1995 were reviewed. Patients were included in the study if they had spinal cord trauma resulting in greater weakness of the upper extremities than of the lower extremities. Patients who did not complete the rehabilitation programme were excluded.

The Department of Rehabilitation Medicine at TTSH is the main spinal rehabilitation centre in the country, where most spinal cord patients requiring inpatient rehabilitation would be admitted. Patients would be screened by a rehabilitation physician prior to admission. Criteria for admission include medical stability and the ability to participate in therapy for at least $2 \mathrm{~h}$ a day. Patients were scored weekly, and team conferences would be held. They would be discharged when they have reached their maximum funtional status.

Data studied included: (1) Injury characteristics: this included mechanism of injury, presence of bony injury, documented cervical spondylosis on radiological studies and surgical procedures performed; (2) Neurologic characteristics: this included admission 
and discharge motor scores (i.e. motor power scores within the first week and on discharge from the rehabilitation centre, using the ASIA criteria), ${ }^{10}$ presence of documented sensory loss on admission, presence of dysaesthetic pain, and significant spasticity of the lower extremities (defined as spasticity requiring oral medications); (3) Bladder management on admission and discharge: this was categorized into indwelling catheterization, intermittent catheterization, reflex voiding and spontaneous voiding; (4) Functional outcome on admission and discharge as measured by the Modified Barthel Index (MBI) was documented at rehabilitation admission and discharge. ${ }^{11}$

\section{Data analysis}

Descriptive statistics were used to characterize the study sample. Student $t$-test was used for comparison of means of continuous variables with normal distributions. Relationships between continuous variables were analyzed using Pearson's test of correlation while that between nominal/ordinal variables were analyzed using the chi-square test. Multiple regression analysis was used to determine variables that may predict discharge functional outcome as defined by the discharge MBI. Statistical significance was set at $P<0.05$.

\section{Results}

\section{Demographic characteristics}

Altogether, there were 73 patients in the study, with a mean age of $53.5 \pm 14.5$ years (range 22-89 years). There were 67 males and six females. Racially, there were 64 Chinese, four Malays, four Indians and one Eurasian.

\section{Injury characteristics}

Falls was the commonest cause of injury in the entire sample - total of 40 falls $(54.8 \%)$, with $80 \%$ resulting from falls on level ground and $20 \%$ falls from heights. There was no significant difference in age between these two groups of patients. Twenty-five patients were involved in motor vehicle accidents and the remaining eight sustained direct blunt injuries. There were no gunshot injuries or stabwounds in the study cohort.

Eleven $(15.1 \%)$ patients had cervical vertebral fractures, and they consisted of four avulsion/chip fractures, five vertebral body fractures, one fracture/ dislocation, and one transverse process fracture. Forty-one patients had documented cervical spondylosis on cervical roentgenograms. Three patients were noted to have ossification of the posterior longitudinal ligament. Patients with cervical spondylosis were significantly older compared to those without (59.1 and 41.3 years respectively, $t$-test, $P<0.001$ ).

Thirteen $(17.8 \%)$ patients underwent surgical decompression - seven had laminectomy, three had laminoplasty and another three underwent anterior cervical decompression and fusion.

\section{Neurologic characteristics}

The mean total ASIA motor score on admission was $54.6 \pm 22.9$ and $76.7 \pm 12.8$ on discharge and this difference was significant $(t$-test, $P<0.001)$. Similar improvements were also noted in the mean admission/ discharge upper and lower limb motor scores (Table 1). Most patients had greater proximal (C5-7) than distal (C8-T1) upper limb scores on admission, with the mean proximal and distal upper limb scores being $57.3 \%$ and $3.6 \%$ of normal respectively.

The admission motor score was significantly correlated with the discharge motor score $(r=0.51$, $P<0.0001)$, admission MBI $(r=0.66, P<0.0001)$ and discharge MBI $(0.38, P<0.005)$.

Fourteen $(19.7 \%)$ patients needed anti-spasticity medications for lower extremity spasticity interfering with function. These patients had lower admission motor scores than those without spasticity (34.5 and 59.1 respectively, $t$-test, $P<0.0001)$. There was however, no significant differences in the discharge motor scores (74.3 and 77.4 respectively, $P=0.424$ ).

The admission and discharge bladder management is described in Table 2, with the number of patients who are catheter-dependent (on intermittent catheterization or indwelling catheter) decreasing from 26 on admission to five on discharge. This difference is statistically significant (chi-square, $P<0.05$ ). Patients who were catheter-dependent on admission also had significantly lower admission motor scores (mean admission motor score 44.5 and 60.3 respectively, $t$ test, $P<0.005)$.

Seventeen patients $(23.3 \%)$ had documented dysaesthetic pain and $45(61.6 \%)$ had sensory levels below which there was impairment to pain and touch, mainly at the lower cervical (C6-8) and T1 levels.

\section{Rehabilitation characteristics}

The mean length of rehabilitation stay was $51.4 \pm 32.4$ days and that in the acute facility was $18.2 \pm 15.3$ days, with a median of 14 days.

The mean admission/discharge MBI scores were $42.9 \pm 29.8$ and $89.2 \pm 11.8$ respectively, and this difference was significant $(t$-test, $P<0.001)$. Similar improvements were seen in the selfcare and mobility categories of the MBI. On admission, $72.6 \%$ were severely dependent (MBI $0-60$ ), 20.5\% moderately dependent (MBI 61-90) and 8.3\% independent (MBI 91-100). Upon discharge, $4.1 \%$ were severely dependent, 39.7\% moderately dependent and 56.2\% independent. Ambulation-wise, 65 patients (89.0\%) needed minimal supervision or were totally independent on discharge compared to 18 patients $(24.6 \%)$ on admission (Table 3). Two patients were wheelchairbound on discharge. Patients with spasticity had both lower admission and discharge MBI scores compared 
Table 1 Mean upper and lower extremity motor at admission and discharge

\begin{tabular}{lcc}
\hline $\begin{array}{l}\text { Mean motor power } \\
\text { (ASIA motor score) }\end{array}$ & $\begin{array}{c}\text { At admission to } \\
\text { rehabilitation }\end{array}$ & $\begin{array}{c}\text { At discharge from } \\
\text { rehabilitation }\end{array}$ \\
\hline Upper limb & $22.8 \pm 11.9$ & $34.0 \pm 7.7$ \\
Lower limb & $31.8 \pm 13.8$ & $42.8 \pm 8.4$ \\
Total motor score & $54.6 \pm 23.0$ & $76.6 \pm 12.9$ \\
\hline
\end{tabular}

Table 2 Bladder management at admission and discharge

\begin{tabular}{lcr}
\hline & $\begin{array}{c}\text { Number on } \\
\text { admission to }\end{array}$ & $\begin{array}{c}\text { Number on } \\
\text { discharge from } \\
\text { Bladder managilitation (\%) }\end{array}$ \\
\hline rehabilitation (\%)
\end{tabular}

Table 3 Modified Barthel scores on admission and discharge

\begin{tabular}{lcc}
\hline MBI scores & $\begin{array}{c}\text { On admission to } \\
\text { rehabilitation }(\%)\end{array}$ & $\begin{array}{c}\text { On discharge from } \\
\text { rehabilitation }(\%)\end{array}$ \\
\hline $\begin{array}{l}\text { Severly dependent } \\
(0-60)\end{array}$ & $53(72.6 \%)$ & $3(4.1 \%)$ \\
$\begin{array}{l}\text { Moderately dependent } \\
(61-90)\end{array}$ & $15(20.5 \%)$ & $29(39.7 \%)$ \\
$\begin{array}{l}\text { Minimally dependent } \\
\text { totally independent } \\
(91-100)\end{array}$ & $5(6.8 \%)$ & $41(56.2 \%)$ \\
\hline
\end{tabular}

to those without (25.9 vs 46.3 for admission MBI and 82.7 vs 90.8 for discharge MBI respectively, $t$-test, $P<0.01$ and $P<0.05$ respectively). There were no significant differences in both the admission and discharge motor and MBI scores between patients who underwent surgical decompression and those who did not $(t$-test, $P<0.05)$.

Multiple regression analysis of clinical factors significantly correlated with the discharge MBI was performed. These included age, spasticity, admission motor score and admission MBI score. Three factors were found to be predictive of discharge MBI scores, namely, admission MBI score $(P<0.001$, beta $=0.177)$ presence of spasticity $(P=0.038$, beta $=-0.221)$ and age $(P=0.006$, beta $=-2.184)$ with adjusted $\mathrm{R}$ square value of 0.331 .

\section{Discussion}

The central cord syndrome as described by Schneider, occurs most commonly in older persons with cervical spondylosis and hyperextension injuries, without apparent damage to the bony spine. ${ }^{4}$ The findings in our study are congruous with Schneider's with regard to the etiology, age, presence of cervical spondylosis and the relatively minor injuries sustained. This is in contradistinction to the recent reports by Roth and Penrod in which they described the 'heterogenous nature' of patients with traumatic central cord syndrome. They described two groups of patients with bimodal age peaks, with different mechanisms of injury and incidence of fractures in the different age groups. The older group were frequently injured in minor falls and have cervical spondylosis, whilst the younger group were injured as a result of major trauma with a higher incidence of cervical fractures, and were lucky enough not to sustain complete injuries as a result of their fractures. 5,6

The incidence of minor falls in our population was relatively high compared to that of the general spinal cord injured population in Singapore whereby minor falls accounted for $8.4 \%$ of all spinal cord injury. ${ }^{12}$ The incidence of motor vehicle accidents is however, quite similar.

Given that cervical spondylosis is a degenerative disorder, it is not surprising that patients in our study with central cord syndrome were significantly older than the rest. The higher risk of patients with cervical spondylosis sustaining the central cord syndrome is believed to be due to the following mechanisms: (1) Gradual compression of the cord, causing deformation of anterior horns of the gray matter as well as the lateral columns; ${ }^{13}$ (2) Narrowing and encroachment of the spinal canal by osteophytes, increasing the risk of cord damage during hyperextension and relatively minor injuries. ${ }^{6}$

In addition, the presence of ossified posterior longitudinal ligament (OPLL) in our patients $(4 \%)$ could further accentuate cord compression. OPLL seems to be a phenomenon which is commoner in Asiatic countries in which it is reported to be found in $2-3 \%$ of cervical roentograms of outpatients, ${ }^{14}$ in comparison to the Caucasian population $(0.2 \%$ at the Mayo clinic and $0.6 \%$ in Hawaii). ${ }^{15}$

Significant gains in motor power were seen from admission to rehabilitation discharge. Those with lower admission motor scores made greater gains in motor power during rehabilitation, although they did not necessarily end up with higher discharge motor scores. This is not unexpected, given that a ceiling effect occurs, with lesser gains made by those with higher admission motor scores. Whether these gains are spontaneous or a result of rehabilitation or both, is not known.

The predominance of upper limb weakness especially those involving muscles of the hand in patients with CCS has been attributed to greater involvement of the most medial fibres of the lateral corticospinal tract which serve the upper limb and hands, ${ }^{16}$ although Nathan and Smith and others in a review of the literature, could find no evidence for such 
lamination. ${ }^{17}$ Hopkins postulates that some segmental organization could take place as the fibres pass medially to their termination, and a cervical medullary lesion could damage a large proportion of corticospinal fibres destined for these segments. ${ }^{18}$

The incidence of pain/hyperpathia documented during admission is comparable to that in Merriam's series $(22.1 \%){ }^{1}$ Interestingly, two patients with hyperpathia also had concomitant reflex sympathetic dystrophy confirmed on bone scan and both had partial resolution of pain after short courses of steroids.

Bladder outcome in patients with CCS have been reported to be better than other patients with incomplete tetraplegia, and despite severe neurological dysfunction during the first few weeks, considerable bladder functional recovery can occur. ${ }^{19}$ This recovery has been reported to occur usually within the first 6 months. ${ }^{19}$ This good outcome is believed to be due to the lesser involvement of the sacral fibres. ${ }^{16,18}$ The incidence of CCS patients achieving spontaneous voiding has been reported to vary from $52-84 \%$., Bladder outcome in our series is considered good as $93.2 \%$ of patients were able to void spontaneously or reflexly upon discharge.

The incidence of spasticity in CCS has been little studied in previous studies. Perkash in his study of 25 patients, found that $48 \%$ had severe spasticity within $3-6$ months of injury. ${ }^{17}$ Our study indicated that almost $20 \%$ of patients needed anti-spasticity medications for lower extremity spasticity. Why some patients should develop significant spasticity and others not, is not known. Given the fact that patients with spasticity had significantly lower admission motor scores, it is possible that these patients may have more severe initial cord damage. Neurological recovery, as assessed by the discharge motor score, is however not significantly different for patients without spasticity.

Functional outcome on discharge is good with $56.2 \%$ requiring minimal supervision or were totally independent in all self-care and mobility tasks. Looking at ambulatory status, $87.7 \%$ of patients were independent or needed only supervision on discharge. Bosch in his series found that functional ambulation was achieved in $59 \%$ of patients with traumatic cervical SCI, ${ }^{2}$ while Roth reported that $70-$ $90 \%$ of patients were independent in completing each of the $14 \mathrm{MBI}$ functional tasks on discharge from rehabilitation. ${ }^{5}$ What is important is that even patients with poor initial functional scores made significant gains post-rehabilitation.

Even though admission Barthel score, age and spasticity were significant factors influencing functional outcome, they only accounted for $33 \%$ of the variance. Age as an adverse prognostic factor on functional outcome has been reported previously. ${ }^{5,6} \mathrm{It}$ has been postulated that elderly patients tend to have higher incidence of cervical spondylosis and cord ischemia from atherosclerotic changes of the vertebral and feeding vessels and this may result in poorer neurological and hence, functional recovery. In addition, elderly patients tend to have a higher incidence of comorbid medical illnesses, and this could have contributed to a worse functional outcome. This factor was however, not examined in our study.

The presence of spasticity as a statistically significant influence on functional outcome has not been well documented previously. It is interesting to note that this significance is independent of the admission Barthel score. Possible adverse effects of spasticity on functional outcome include decreased motor control secondary to co-contraction of muscles, impaired co-ordination and excessive spasms/mass movements which can impair both balance (sitting and standing) and ambulation. In our study, the two patients who were wheelchair-bound had severe uncontrolled spasticity despite medications. Perkash also noted that $48 \%$ of patients in his study failed to ambulate because of severe spasticity. ${ }^{19}$

A limitation in this study is the variability in the time of admission to the rehabilitation facility. The initial admission motor scores can therefore not be compared to other studies which score these patients within $1-2$ weeks of injury. ${ }^{1}$ Nevertheless, admission motor scores have been shown to improve during rehabilitation.

Another limitation of this retrospective study is the lack of long-term follow-up of the patient's neurological and functional status. It is certainly possible that further improvements in the above are made postdischarge. Despite this, we conclude that patients with central cord syndrome generally have a good prognosis neurologically and functionally post-rehabilitation.

\section{References}

1 Merriam SF, Taylor TKF, Ruff SJ, Mc Phail MJ. A reappraisal of acute traumatic central cord syndrome. J Bone Joint Surg 1986; 68B: $708-713$.

2 Bosch A, Stauffer ES, Nickel VL. Incomplete traumatic quadriplegia. A ten year review. JAMA 1971; 216: 473 - 478 .

3 Schneider RC, Cherry G, Pantek H. The syndrome of acute central cervical spinal cord injury, with special reference to the mechanics involved in hyperextension injuries of the cervical spine. J Neurosurg 1954; 11: 546-577.

4 Taylor AR, Blackwood W. Paraplegia in hyperextension cervical injuries with normal radiographic appearances. J Bone Joint Surg 1948; 30-B: $245-248$.

5 Roth EJ, Lawler MH, Yarkony GM. Traumatic Central Cord syndrome: Clinical features and functional outcomes. Arch Phys Med Rehabil 1990; 71: $18-23$

6 Penrod LE, Hegde SK, Ditunno JF. Age effect on prognosis for functional recovery in acute, traumatic central cord syndrome. Arch Phys Med Rehabil 1990; 71: 963 -968.

7 Taylor AR. The mechanism of injury to the spinal cord in the neck without damage to the vertebral column. J Bone and Joint Surg 1951; 33B: $543-547$.

8 Rand RW, Crandall PH. Central spinal cord syndrome in hyperextension injuries of the cervical spine. J Bone Joint Surg 1962; 44A: $1415-1422$.

9 Shrosbree RD. Acute central cervical spinal cord syndrome: aetiology, age incidence and relationship to the orthopaedic injury. Paraplegia 1977; 14: 251 - 258 
10 American Spinal Injury Association. Standards for neurological classification of spinal injury patients. Chicago: American Spinal Injury Association, 1987.

11 Shah S, Varclay F, Cooper B. Improving the sensitivity of the Barthel Index for stroke rehabilitation. J Clin Epidemiol 1989; 42: $703-709$.

12 Tan ES, Balachandran N. The causes, pattern and effects of spinal injury in Singapore. Clin Rehabilitation 1987: 1: 101-106.

13 Breig A, Turnbull I, Hasler O. Effects of mechanical stresses on the spinal cord in cervical spondylosis: a study on fresh cadaver material. J Neurosurg 1966; 25: $45-56$.

14 Hirabayashi K, Satomi K, Sasaki T. Ossification of the Posterior Longitudinal Ligament in the Cervical Spine. In: The cervical spine research society editorial committee: Sherk $\mathrm{HH}$ (ed.) The cervical spine. JB Lippincott company, 1989; pp 678-692.
15 Tsuyama N. Ossification of the posterior longitudinal ligament of the spine. Clin Orthop 1984; 184: 71 .

16 Foerster O. Symptomatologie der Erkrankungen des Ruckenmarks and seiner Wurzeln. Bumke u. Foersters Handb Neurol 1936; 5: $1-403$.

17 Nathan PW, Smith MC. Long descending tracts in man. 1. Review of present knowledge. Brain 1955; 78: $248-303$.

18 Hopkins A, Rudge P. Hyperpathia in the central cervical cord syndrome. J Neurol Neurosurg and Psychiatry 1973; 36: 637-642.

19 Perkash I. Management of neurogenic bladder by dysfunctions following acute traumatic cervical central cord syndrome (incomplete tetraplegia). Paraplegia 1977-78; 15: 21-37. 